\title{
Vibration of an Incompressible viscoelastic shell of rate type
}

\author{
Angiolo Farina, Lorenzo Fusi, Fabio Rosso and Giuseppe Saccomandi
}

\begin{abstract}
We study the oscillations of a spherical shell of a rate-type viscoelastic solid subject to a pressure difference between the inner and the outer surface. The stable equilibrium configurations, in the class of spherically symmetric deformations, correspond to the minima of the elastic energy function. Numerical simulations indicate that the way in which the equilibrium state is reached, strongly depends on the material parameters.
\end{abstract}

Mathematics Subject Classification. 74A99, 74D99.

Keywords. Viscoelastic materials, Constitutive models, Frame indifferent derivative, Spherically symmetric deformations.

\section{Introduction}

Instabilities of inflated spherical membranes are a stimulating problem in continuum mechanics (see, e.g., Chap. 7 in [1]). The ordinary differential equations of motion describing the finite amplitude of radial oscillations of thick-walled hyperelastic spherical shells are well known, see Truesdell and Noll [2] (pages. 217-219) for a derivation of such equations and for references to the original literature. The same problem has been studied by Calderer [3] in the framework of the special theory of viscoelasticity. In this paper, the author started with a general elastic strain-energy density $W(\mathbb{F})$ where $\mathbb{F}$ is the gradient of the deformation and, adding to $W$ an extra history-type term, i.e., a term which depends via a suitable integral on the history of $\mathbb{F}$ up to time $t$, she introduced a free energy functional to describe the mechanical behavior of the material. In [3], the author provided a rigorous proof of the possibility of finite-time blow-up of the solutions. The existence of singular solutions in such class of materials has been also considered in [4] in the framework of traveling waves. A model similar to the one proposed in [3] has also been considered by Fosdick and $\mathrm{Yu}[5]$. In this case, the authors studied the stability of the radial oscillations of a sphere via a suitable Lyapunov functional.

One of the many applications of this basic problem is bioenteric intragastric balloons for the treatment of obesity [6]. Recently, there has been a renewed interest on this problem [7] studied using the celebrated quasi-linear viscoelastic model proposed by Pipkin and Rogers [8]. However, within the framework of traveling waves, we do know that global solutions may not exist for the quasi-linear viscoelastic models (see, e.g., [9]).

In the present article, the spherical vibrations of a shell are deemed in the framework of rate-type viscoelastic materials. The model we investigate is a nonlinear generalization of the standard linear solid. The standard linear solid is a quite versatile model of linear elasticity because it describes both phenomena as creep and stress relaxation on the basis of a simple setting. A possible generalization of this model to a three-dimensional nonlinear context has been first put forward some years ago in $[10,11]$. In the three-dimensional generalization, one major problem is the choice of the objective derivative required to describe stress relaxation. Indeed, this choice is not unique. The differences associated with the possible choices of the objective derivative have been pointed out in [12] and in [13]. 
In this paper, to simplify the algebra, the material parameters are set constant. However, as far as the elastic parameters are concerned, this hypothesis is not strictly necessary and many of our results might be extended to a more general nonlinear setting. Also, the viscosity, i.e., the parameter regulating the dissipative part of the Cauchy stress, is supposed to be constant. Indeed, a nonlinear viscous dissipation could not only add further technical complexity, but also the mathematical problem may turn out to be ill posed, unless the function expressing the viscosity in terms of the shear rate or the Cauchy-Green tensor obeys very restrictive hypotheses [14].

The paper is organized as follows: in Sect. 2, we illustrate the constitutive model and, in Sects. 3 and 4 , we investigate the radial deformations of a spherical shell. Next, in Sect. 5 , we focus on the equilibrium configurations confining ourselves to the class of spherical disturbances. The system dynamics is analyzed in Sect. 6 . A few concluding remarks are drawn in the last section.

\section{Preliminaries}

Here, we recall some standard notations used in Continuum Mechanics (see, e. g., [15, 16]). Let

$$
\boldsymbol{x}=\chi(\boldsymbol{X}, t)
$$

be the deformation of a three-dimensional body in the Euclidean space, where $\boldsymbol{x}$ and $\boldsymbol{X}$ are the respective positions of a generic particle of the body at time $t$ and at a given initial time $t_{o}$. Then,

$$
\mathbb{F}:=\frac{\partial \boldsymbol{\chi}(\boldsymbol{X}, t)}{\partial \boldsymbol{X}}, \quad \mathbb{B}:=\mathbb{F}^{\mathrm{T}}, \quad \mathbb{L}:=\dot{F}^{-1}
$$

define, respectively, the deformation gradient, the Cauchy-Green deformation tensor, and the spatial velocity gradient tensor. The superimposed dot identifies the usual material time derivative. The symmetric tensor

$$
\mathbb{D}:=\frac{1}{2}\left(\mathbb{L}+\mathbb{L}^{\mathrm{T}}\right),
$$

is the stretching tensor. Next, we assume that the material is mechanically incompressible, i.e.,

$$
\operatorname{det} \mathbb{F}=1 \text {, }
$$

which entails the introduction of an unknown tensor $-p \mathbb{I}$ (see [17]).

According to the results illustrated in the recent paper [13], we consider the following constitutive model $[18,19]$

$$
\left\{\begin{array}{l}
\mathbb{T}=-p \mathbb{I}+\beta_{1} \mathbb{B}+\beta_{-1} \mathbb{B}^{-1}+\mathbb{T}^{V} \\
\dot{\mathbb{T}}^{V}-\frac{1}{2}\left(\mathbb{T}^{V} \mathbb{L}^{\mathrm{T}}+\mathbb{L}^{V}\right)+\frac{1}{2}\left(\mathbb{T}^{V} \mathbb{L}+\mathbb{L}^{\mathrm{T}} \mathbb{T}^{V}\right)=-\phi\left[\mathbb{T}^{V}-2 \eta \mathbb{D},\right.
\end{array}\right.
$$

where $\beta_{1}, \beta_{-1}$, are, in general, material functions of the invariants of $\mathbb{B}$, while $\eta$ and $\phi$ are constant parameters representing the material viscosity and a characteristic time ${ }^{1}$, respectively. In particular, we take $\phi$ and $\eta$ non-negative. Concerning $\beta_{1}$ and $\beta_{-1}$, we consider them as constant parameters and set

$$
\beta_{1}=\frac{G}{1+\alpha}, \quad \beta_{-1}=-\frac{\alpha G}{1+\alpha},
$$

with $G=\left(\beta_{1}-\beta_{-1}\right)>0$, material elastic modulus and $\alpha \in[0,1]$. When $\alpha=0$, we recover the neoHookean case.

\footnotetext{
${ }^{1}$ The parameter $\phi$ is essentially the retardation time.
} 


\section{Radial deformation and stress}

We consider a spherical shell whose internal and external radii in the undeformed configuration (which we assume to be the reference configuration) are $S$ and $\Sigma$, respectively. We then consider a set of curvilinear coordinates of spherical type and denote by $(R, \Theta, \Phi)$ the material coordinates and by $(r, \theta, \varphi)$ the spatial ones. We confine ourselves only to spherically symmetric deformations. So, we represent (1) as

$$
r=r(R, t), \quad \Theta=\theta, \quad \Phi=\varphi,
$$

and, recalling (4), we obtain ${ }^{2}$ (see also [20])

$$
r=r(R, t)=\sqrt[3]{\left(R^{3}-S^{3}\right)+s^{3}(t)}, \quad \text { with } \quad S \leq R \leq \Sigma,
$$

where $s(t)$ is the position at time $t$ of the shell inner radius. The position at time $t$ of the outer radius is

$$
\sigma(t)=\sqrt[3]{\Sigma^{3}-S^{3}+s^{3}(t)}
$$

The inverse of $(8)$ is

$$
R(r, t)=\sqrt[3]{S^{3}+\left(r^{3}-s^{3}(t)\right)}, \quad \text { with } \quad s(t) \leq r \leq \sigma(t) .
$$

Hence, $s(t)$ and $\sigma(t)$ are the two boundaries delimiting the material domain

$$
\Omega=\{s(t) \leq r \leq \sigma(t),-\pi / 2<\theta<\pi / 2,-\pi<\varphi \leq \pi\},
$$

We remark that $\Omega$ is a time evolving domain, since both $s(t)$ and $\sigma(t)$ evolve in time. Actually $s(t)$ and, due to formula (9), also $\sigma(t)$ are free boundaries since their evolution is a priori unknown.

Denoting by $\dot{r}(R, t)$ and $\ddot{r}(R, t)$ the radial velocity and acceleration, the Eulerian descriptions of these fields are

$$
\begin{aligned}
& v(r, t)=\left.\dot{r}(R, t)\right|_{R=R(r, t)}=\frac{s^{2} \dot{s}}{r^{2}}, \quad s(t) \leq r \leq \sigma(t), \\
&\left.\ddot{r}(R, t)\right|_{R=R(r, t)}=\frac{\partial v}{\partial t}+v \frac{\partial v}{\partial r}=\frac{\ddot{s} s^{2}+2 \dot{s}^{2} s}{r^{2}}-2 \frac{\left(\dot{s} s^{2}\right)^{2}}{r^{5}}, \quad s(t) \leq r \leq \sigma(t) .
\end{aligned}
$$

In particular, since $\boldsymbol{v}=v(r, t) \boldsymbol{e}_{r}$, it is trivial to verify that $\nabla \cdot \boldsymbol{v}=0$. Moreover, introducing the so-called acceleration potential $\zeta$, such that $\ddot{r}=-\frac{\partial \zeta}{\partial r}$, we have

$$
\zeta=\frac{\ddot{s} s^{2}+2 \dot{s}^{2} s}{r}-\frac{\left(\dot{s} s^{2}\right)^{2}}{2 r^{4}}
$$

Next, it turns out that

$$
\begin{aligned}
& \mathbb{F}=\left(\begin{array}{ccc}
R^{2} / r^{2} & 0 & 0 \\
0 & r / R & 0 \\
0 & 0 & r / R
\end{array}\right), \quad \mathbb{F}^{-1}=\left(\begin{array}{ccc}
r^{2} / R^{2} & 0 & 0 \\
0 & R / r & 0 \\
0 & 0 & R / r
\end{array}\right) \\
& \mathbb{B}=\left(\begin{array}{ccc}
R^{4} / r^{4} & 0 & 0 \\
0 & r^{2} / R^{2} & 0 \\
0 & 0 & r^{2} / R^{2}
\end{array}\right), \quad \mathbb{B}^{-1}=\left(\begin{array}{ccc}
r^{4} / R^{4} & 0 & 0 \\
0 & R^{2} / r^{2} & 0 \\
0 & 0 & R^{2} / r^{2}
\end{array}\right) \\
& \mathbb{L}=\frac{s^{2} \dot{s}}{r^{3}}\left(\begin{array}{ccc}
-2 & 0 & 0 \\
0 & 1 & 0 \\
0 & 0 & 1
\end{array}\right), \quad \mathbb{D}=\frac{1}{2}\left(\mathbb{L}+\mathbb{L}^{\mathrm{T}}\right)=\mathbb{L} .
\end{aligned}
$$

\footnotetext{
${ }^{2}$ Following $\S 61$ and $\S 62$ in $[2], r^{3}=R^{3}+A(t)$, so that in our notation $A(t)=s^{3}(t)-S^{3}$.
} 
Within the class of spherical deformations, the constitutive Eq. (5) gives rise to the following system

$$
\left\{\begin{array}{l}
T_{r r}=-p+\beta_{1} \frac{R^{4}}{r^{4}}+\beta_{-1} \frac{r^{4}}{R^{4}}+T_{r r}^{V} \\
T_{\theta \theta}=-p+\beta_{1} \frac{r^{2}}{R^{2}}+\beta_{-1} \frac{R^{2}}{r^{2}}+T_{\theta \theta}^{V} \\
T_{r r}^{V}=-\frac{1}{\phi} \dot{T}_{r r}^{V}-4 \eta \frac{s^{2} \dot{s}}{r^{3}} \\
T_{\theta \theta}^{V}=-\frac{1}{\phi} \dot{T}_{\theta \theta}^{V}+2 \eta \frac{s^{2} \dot{s}}{r^{3}}
\end{array}\right.
$$

where the equations for $T_{\varphi \varphi}$ and $T_{\varphi \varphi}^{V}$ are formally identical to those for $T_{\theta \theta}$ and $T_{\theta \theta}^{V}$. This implies that identical initial conditions lead to the same solution. Therefore, assuming $T_{\varphi \varphi}(0)=T_{\theta \theta}(0), T_{\varphi \varphi}$ and $T_{\theta \theta}$ identify.

\section{Motion of the spherical shell}

The only non-trivial component of the motion equation is the radial one, namely

$$
-\rho \frac{\partial \zeta}{\partial r}=\frac{1}{r^{2}} \frac{\partial}{\partial r}\left(r^{2} T_{r r}\right)-\frac{T_{\theta \theta}+T_{\varphi \varphi}}{r},
$$

in which $\rho$ is the material density, body forces are neglected, and $\zeta$ is the acceleration potential (13). Concerning the force driving the motion, we assume that the shell is subjected to a uniform internal pressure $p_{i n}$ and to a uniform pressure $p_{\text {ext }}$ acting on the shell external face. Recalling (12) and that $T_{\theta \theta}(0)=T_{\varphi \varphi}(0)$, Eq. (18) rewrites as

$$
-\rho \frac{\partial \zeta}{\partial r}=\frac{\partial T_{r r}}{\partial r}+\frac{2}{r}\left(T_{r r}-T_{\theta \theta}\right) .
$$

Recalling (17), the last term can be written as

$$
T_{r r}-T_{\theta \theta}=\beta_{1}\left(\frac{R^{4}}{r^{4}}-\frac{r^{2}}{R^{2}}\right)+\beta_{-1}\left(\frac{r^{4}}{R^{4}}-\frac{R^{2}}{r^{2}}\right)+k,
$$

with

$$
k=T_{r r}^{V}-T_{\theta \theta}^{V} .
$$

In particular, exploiting $(17)_{2}$ and $(17)_{4}$, we have

whose solution is

$$
\dot{k}+\phi k=-6 \eta \phi \frac{s^{2} \dot{s}}{r^{3}},
$$

$$
k=k_{0} e^{-\phi t}-\frac{6 \eta \phi}{r^{3}} \int_{0}^{t} \dot{s}(\tau) s^{2}(\tau) e^{-\phi(t-\tau)} \mathrm{d} \tau,
$$

where $k_{0}=\left.k\right|_{t=0}$. We now insert (22) into (20) and integrate (19) in the $r$ variable, for $r \in[s, \sigma]$. Considering the various terms separately, we have 


$$
\begin{gathered}
-\int_{s}^{\sigma} \frac{\partial \zeta}{\partial r} \mathrm{~d} r=\zeta(s)-\zeta(\sigma)=\left(\ddot{s} s+2 \dot{s}^{2}\right) \frac{\sigma-s}{\sigma}+\frac{\dot{s}^{2}}{2}\left(\frac{s^{4}-\sigma^{4}}{\sigma^{4}}\right), \\
\int_{s}^{\sigma} \frac{\partial T_{r r}}{\partial r} \mathrm{~d} r=\Delta p, \quad \text { with } \quad \Delta p(t)=p_{i n}(t)-p_{\text {ext }}(t), \\
\int_{s}^{\sigma} \frac{2}{r}\left(T_{r r}-T_{\theta \theta}\right) \mathrm{d} r=f+2 \int_{s}^{\sigma} \frac{k}{r} \mathrm{~d} r,
\end{gathered}
$$

with

$$
f=2 \int_{s}^{\sigma}\left[\beta_{1}\left(\frac{R^{4}}{r^{4}}-\frac{r^{2}}{R^{2}}\right)+\beta_{-1}\left(\frac{r^{4}}{R^{4}}-\frac{R^{2}}{r^{2}}\right)\right] \frac{\mathrm{d} r}{r} .
$$

So, focussing on (23), we evaluate the second integral getting

$$
\begin{aligned}
2 \int_{s}^{\sigma} \frac{k}{r} \mathrm{~d} r & =2 k_{0} e^{-\phi t} \ln \left(\frac{\sigma}{s}\right)+4 \eta \phi\left(\frac{1}{\sigma^{3}}-\frac{1}{s^{3}}\right) y(t) \\
& =2 k_{0} e^{-\phi t} \ln \left(\frac{\sigma}{s}\right)-4 \eta \phi \frac{\Sigma^{3}-S^{3}}{\sigma^{3} s^{3}} y(t),
\end{aligned}
$$

where

$$
y(t)=\int_{0}^{t} \dot{s}(\tau) s^{2}(\tau) e^{-\phi(t-\tau)} \mathrm{d} \tau .
$$

Concerning the term $f$ given by (24), we introduce

$$
\xi=\frac{R}{r}, \underset{(10)}{\Rightarrow}\left\{\begin{array}{l}
\frac{\mathrm{d} r}{r}=\frac{\xi^{2}}{1-\xi^{3}} \mathrm{~d} \xi \\
\left.\xi\right|_{s}=\frac{S}{s},\left.\quad \xi\right|_{\sigma}=\frac{\Sigma}{\sigma},
\end{array}\right.
$$

so that

$$
\begin{aligned}
f= & -\frac{G}{1+\alpha}\left\{2\left(\frac{\Sigma}{\sigma}-\frac{S}{s}\right)+\frac{1}{2}\left(\left(\frac{\Sigma}{\sigma}\right)^{4}-\left(\frac{S}{s}\right)^{4}\right)+\right. \\
& \left.+2 \alpha\left(\frac{s}{S}-\frac{\sigma}{\Sigma}\right)+\alpha\left(\left(\frac{\Sigma}{\sigma}\right)^{2}-\left(\frac{S}{s}\right)^{2}\right)\right\}
\end{aligned}
$$

where we have exploited (6).

Summarizing, after the integration, the motion equation rewrites as

$$
\begin{aligned}
\rho\left[\left(\ddot{s} s+2 \dot{s}^{2}\right) \frac{\sigma-s}{\sigma}+\frac{\dot{s}^{2}}{2}\left(\frac{s^{4}-\sigma^{4}}{\sigma^{4}}\right)\right]= & \Delta p+2 k_{0} e^{-\phi t} \ln \left(\frac{\sigma}{s}\right)+ \\
& -4 \eta \phi \frac{\Sigma^{3}-S^{3}}{\sigma^{3} s^{3}} y(t)+f(s),
\end{aligned}
$$

with $y$ and $f$ given by (26) and (27), respectively.

We now introduce the characteristic dimensionless parameters

$$
\tilde{\Sigma}=\frac{\Sigma}{S}, \quad \tilde{a}=\frac{\eta \phi}{G}, \quad \tilde{b}=\phi t_{c}, \quad \text { with } t_{c}=\sqrt{\frac{\rho S^{2}}{G}},
$$


the dimensionless variables

$$
\tilde{s}=\frac{s}{S}, \quad \tilde{\sigma}=\frac{\sigma}{S}=\sqrt[3]{\tilde{\Sigma}^{3}-1+\tilde{s}^{3}}, \quad \tilde{t}=\frac{t}{t_{c}}
$$

and the functions

$$
\begin{gathered}
\tilde{f}=\frac{f}{G}=-\frac{1}{1+\alpha}\left\{2\left(\frac{\tilde{\Sigma}}{\tilde{\sigma}}-\frac{1}{\tilde{s}}\right)+\frac{1}{2}\left(\left(\frac{\tilde{\Sigma}}{\tilde{\sigma}}\right)^{4}-\frac{1}{\tilde{s}^{4}}\right)\right. \\
\left.+2 \alpha\left(\tilde{s}-\frac{\tilde{\sigma}}{\tilde{\Sigma}}\right)+\alpha\left(\left(\frac{\tilde{\Sigma}}{\tilde{\sigma}}\right)^{2}-\frac{1}{\tilde{s}^{2}}\right)\right\}, \\
\tilde{y}=\frac{y}{S^{3}}=e^{-b \tilde{t}} \int_{0}^{\tilde{t}} \frac{\mathrm{d} \tilde{s}}{\mathrm{~d} \tilde{\tau}} \tilde{s}^{2}(\tilde{\tau}) e^{b \tilde{\tau}} d \tilde{\tau}, \\
\Delta \tilde{p}(\tilde{t})=\frac{\Delta p\left(\tilde{t} t_{c}\right)}{G} .
\end{gathered}
$$

We thus obtain the dimensionless equation (where we omit " $"$ to keep the notation as light as possible)

$$
\begin{aligned}
\ddot{s} s\left(1-\frac{s}{\sigma}\right)+\dot{s}^{2}\left(\frac{3}{2}-2 \frac{s}{\sigma}+\frac{1}{2} \frac{s^{4}}{\sigma^{4}}\right)= & \Delta p+2 \frac{k_{0}}{G} e^{-b t} \ln \left(\frac{\sigma}{s}\right)+ \\
& -4 a \frac{\Sigma^{3}-1}{\sigma^{3} s^{3}} y+f .
\end{aligned}
$$

If we consider as initial condition $s(0)=1$, i.e., the undeformed configuration, we have $k_{0}=0$, and we can transform Eq. ( 31 ) in a system of two autonomous ODEs

$$
\left\{\begin{array}{l}
\ddot{s} s\left(1-\frac{s}{\sigma}\right)+\dot{s}^{2}\left(\frac{3}{2}-2 \frac{s}{\sigma}+\frac{1}{2} \frac{s^{4}}{\sigma^{4}}\right)=\Delta p-4 a \frac{\Sigma^{3}-1}{\sigma^{3} s^{3}} y+f, \\
\dot{y}=-b y+\dot{s} s^{2}
\end{array}\right.
$$

whose initial conditions are $s(0)=1, \dot{s}(0)=\dot{s}_{o}, y(0)=0$. In Sect. 5 , we shall analyze the stability of the equilibrium configurations of (32) in the class of spherically symmetric disturbances.

\section{Equilibrium configurations}

In this section, we focus on the equilibrium configurations of system (32) and their stability. However, it should be stressed that our analysis does not guarantee stability to arbitrary disturbances, since we only consider a restricted class of deformations, i.e., the spherically symmetric ones. A similar issue has been deeply investigated in $[21,22]$. In these papers, the authors, studying the flow between rotating plates, showed the existence of an infinite number of solutions that lack axial symmetry close to any axially symmetric solution. Thus, none of the axially symmetric solutions are stable within the full class of possible solutions, while just restricting to axially symmetric class one finds such solutions to be stable. This general conclusion obviously applies also to our case.

We start considering a prescribed constant pressure difference $\Delta p$ between inlet and outlet, and $k_{0}=0$, so that system (32) is autonomous. Recalling the energy balance, we have

$$
\frac{d}{d t}\left(\int_{\Omega} \rho \frac{v^{2}}{2} d^{3} x\right)=-\int_{\Omega} \mathbb{T} \cdot \mathbb{D} d^{3} x+\int_{\partial \Omega} \boldsymbol{T} \cdot v \boldsymbol{e}_{r} d s
$$


where $\Omega$ is given by (11), $v$ is the Eulerian velocity given by (12) and $\boldsymbol{T}$ represents the force (per unit surface) acting on the shell boundary, namely

$$
\int_{\partial \Omega} \boldsymbol{T} \cdot v \boldsymbol{e}_{r} d s=4 \pi \Delta p \dot{s} s^{2} .
$$

The last term at the right hand side of (33) becomes

$$
\int_{\Omega} \rho \frac{v^{2}}{2} d^{3} x=4 \pi \rho\left[\frac{\dot{s}^{2} s^{3}}{2}\left(1-\frac{s}{\sigma}\right)\right],
$$

while, recalling (16), (17), and (21),

$$
-\int_{\Omega} \mathbb{T} \cdot \mathbb{D} d^{3} x=4 \pi \dot{s} s^{2} f(s)+4 \pi \int_{s}^{\sigma} 2 \frac{k(r, t)}{r} \dot{s} s^{2} \mathrm{~d} r,
$$

with $f$ given by (24) or, in an explicit form, by (27). Thus, recalling (25), (26) and the dimensionless quantities introduced in (28) and (29), formula (33) can be rewritten $\mathrm{as}^{3}$

$$
\frac{d}{d t}\left[\frac{A(s)}{2} \dot{s}^{2}+V(s)\right]=-\chi y M(s),
$$

where

$$
\begin{array}{r}
V(s)=-\frac{s^{3} \Delta p}{3}-\int_{1}^{s} z^{2} f \mathrm{~d} z, \\
A(s)=s^{3}\left(1-\frac{s}{\sigma}\right), \\
M(s)=\frac{1}{s \sigma^{3}(s)}, \quad \chi=4 a\left(\Sigma^{3}-1\right)>0,
\end{array}
$$

where both $A(s)$ and $M(s)$ are strictly positive. So, the equilibrium configurations $\left(s_{\mathrm{eq}}, y_{\mathrm{eq}}\right)$ of $(32)$, which are given by

$$
\left\{\begin{array}{l}
\Delta p-4 a \frac{\Sigma^{3}-1}{\sigma^{3} s_{\mathrm{eq}}^{3}} y_{\mathrm{eq}}+f\left(s_{\mathrm{eq}}\right)=0 \\
y_{\mathrm{eq}}=0
\end{array}\right.
$$

can be obtained by solving

$$
V^{\prime}(s)=0, \Rightarrow f(s)=-\Delta p,
$$

with $V(s)$ given by (35). So, in this specific case, the equilibrium configuration $s_{\text {eq }}$ corresponds to the stationary points of $V(s)$. This result should not be misleading, as it is due to the peculiar mathematical structure of the problem. Indeed, since $s_{\text {eq }}$ is a stationary point of $V$, it could be automatically assumed that $s_{\text {eq }}$ is stable if it is an isolated minimum of $V$. We, however, remark that, on dissipative systems, one cannot make general conclusions concerning stability just on the basis of the stored energy. In fact, in entropy producing systems, it is the dissipation (entropy production) that governs the evolution of the problem. For this reason, we study the stability of $s_{\text {eq }}$ through a linear analysis. In other words, we

\footnotetext{
${ }^{3}$ We remark that we could have obtained (34) just by multiplying ( 32$)_{1}$ by $\dot{s} s^{2}$.
} 

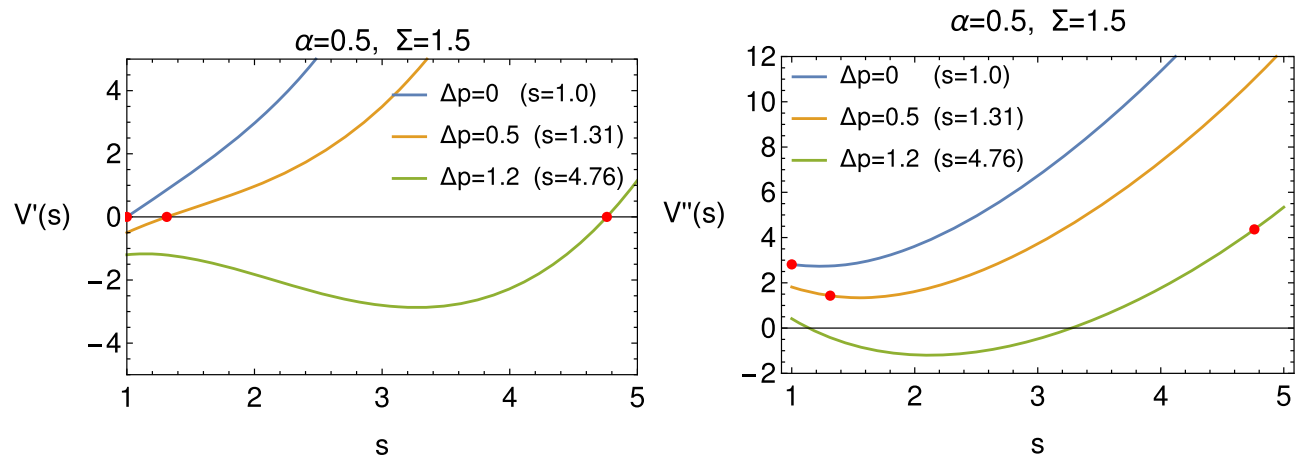

FIG. 1. Stable equilibria corresponding to three different values of $\Delta p$ for fixed choices of $\alpha$ and $\Sigma$. We notice that $s_{\text {eq }}$ corresponding to $\Delta p=0$ is the undeformed one, which, by the way, is also stable

analyze the stability of $s_{\text {eq }}$, i.e., the generic solution of (36), writing (32) as a first-order system in the unknowns $(s, u, y)$, with $u=\dot{s}$, i.e.,

$$
\left\{\begin{array}{l}
\dot{s}=u, \\
\dot{u}=-\frac{1}{A(S)}\left[\frac{A^{\prime}}{2} u^{2}+V^{\prime}+\chi y M\right], \\
\dot{y}=-b y+u s^{2},
\end{array}\right.
$$

and performing a linear analysis around $\left(s_{\text {eq }}, 0,0\right)$. This means, as already remarked, that this analysis provides information only within the class of spherically symmetric deformations. Evaluating the Jacobi matrix in $\left(s_{\mathrm{eq}}, 0,0\right)$, we have

$$
\mathbb{J}\left(s_{\mathrm{eq}}, 0,0\right)=\left(\begin{array}{ccc}
0 & 1 & 0 \\
J_{21} & 0 & J_{23} \\
0 & s_{\mathrm{eq}}^{2} & -b
\end{array}\right),
$$

where

$$
J_{21}\left(s_{\mathrm{eq}}, 0,0\right)=-\frac{V^{\prime \prime}\left(s_{\mathrm{eq}}\right)}{A\left(s_{\mathrm{eq}}\right)}, \quad J_{23}\left(s_{\mathrm{eq}}, 0,0\right)=-\chi \frac{M\left(s_{\mathrm{eq}}\right)}{A\left(s_{\mathrm{eq}}\right)}<0,
$$

The corresponding characteristic polynomial is

$$
\lambda^{3}+b \lambda^{2}-\lambda\left(J_{21}+J_{23} s_{\text {eq }}^{2}\right)-b J_{21}=0 .
$$

If all the eigenvalues satisfy $\operatorname{Re}(\lambda)<0$, then $\left(s_{\text {eq }}, 0,0\right)$ is locally asymptotically stable. In particular, if $s_{\text {eq }}$ is an isolated minimum of $V$, i.e., $V^{\prime \prime}\left(s_{\mathrm{eq}}\right)>0$, the coefficients of $(37)$ are positive. Hence, applying the Routh-Horwitz criterion [23,24], we find that all roots of $(37)$ have negative real part, and so $\left(s_{\text {eq }}, 0,0\right)$ is stable. In particular, the natural undeformed configuration $(1,0,0)$, that corresponds to $\Delta p=0$, is stable, see Fig. 1.

On the other hand, if $s_{\text {eq }}$ is an isolated maximum of $V$, i.e., $V^{\prime \prime}\left(s_{\text {eq }}\right)<0$, Routh-Horwitz criterion entails that at least one eigenvalue has positive real part. Hence, the maxima of $V$ correspond to unstable configurations.

Now, still keeping $k_{0}=0$, we investigate the solution to (36) corresponding to a prescribed $\Delta p>0$. Figure 2 shows that, depending on $\alpha \in(0,1)$, we can have from one to three equilibria $s_{\text {eq }}$ for given $\Delta p>0$ and $\Sigma>1$.

The typical equilibrium configurations are summarized in Fig. 2. We verified the stability properties by evaluating the sign of $V^{\prime \prime}$. Referring to the upper left panel, we have three equilibrium configurations for $\alpha \in\left(0, \alpha_{\mathrm{cr}}\right)$. However, only two branches are stable. The value $\alpha=\alpha_{1, \mathrm{cr}}$ corresponds to a turning point (the number of equilibria changes). When $\alpha \in\left(\alpha_{\mathrm{cr}}, 1\right)$, only one equilibrium configuration (close to 1$)$ is 

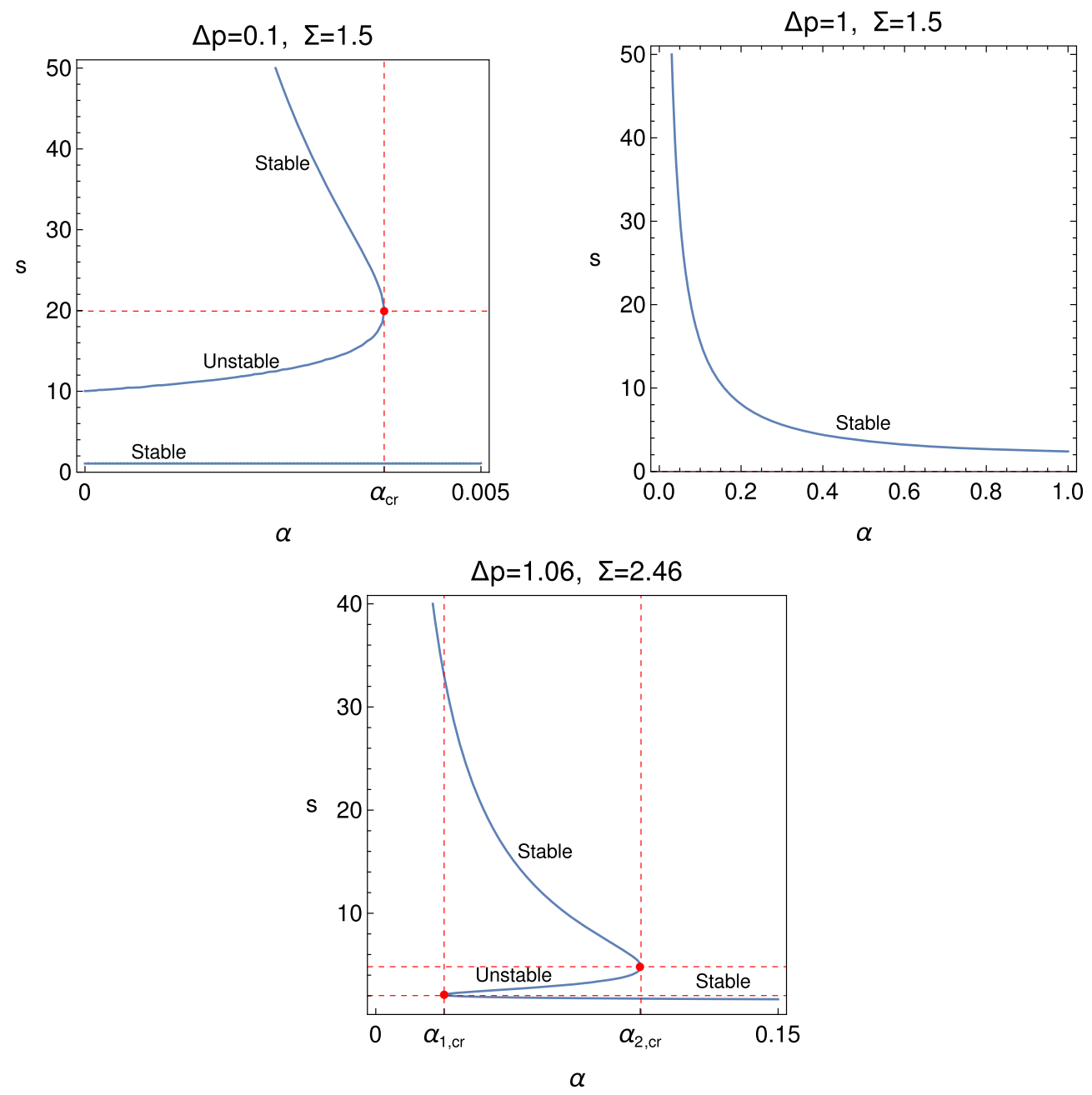

FIG. 2. Depending on the values of $(\Delta p, \Sigma)$, the locus of equilibria shows a single branch or two disjoint branches. In the upper left panel, we have one turning point, $P=\left(\alpha_{\mathrm{cr}}, s_{\mathrm{cr}}\right)=(0.0038,19.924)$, while the upper right panel does not show any turning point. In the lowest panel, we have two turning points, $P_{1}=\left(\alpha_{1, \mathrm{cr}}, s_{1, \mathrm{cr}}\right)=(0.028,2.02), P_{2}=\left(\alpha_{1, \mathrm{cr}}, s_{2, \mathrm{cr}}\right)=$ $(0.0988,4.806)$. The stable/unstable character of equilibria is clearly indicated (see also Fig. 4 in the sequel)

observable. In the upper right panel of Fig. 2, we have a single equilibrium curve which is always stable. In the lower third panel of Fig. 2, we have a single equilibrium curve which shows two turning points $\alpha_{\mathrm{i}, \mathrm{cr}}(i=1,2)$. When $\alpha \in\left(0, \alpha_{1, \mathrm{cr}}\right)$, only one equilibrium stable configuration is observable. However, we remark that the corresponding $s_{\text {eq }}$ values are in an nonphysical range. When $\alpha \in\left(\alpha_{1, \mathrm{cr}}, \alpha_{2, \mathrm{cr}}\right)$, only the lowest and the uppest branches are stable. Finally, for $\alpha \in\left(\alpha_{2, \mathrm{cr}}, 1\right)$, there is only one stable configuration.

Figure 3 shows some examples of stable solutions originated in the attraction basin of equilibria.

We now consider again the lower panel in Fig. 2 and fix an initial value $s_{\mathrm{o}}$ in the interval $\left(s_{1, \mathrm{cr}}, s_{2, \mathrm{cr}}\right)$ and select three different materials, corresponding to three different choices of $\alpha$, namely $s(0)=4$, and

$$
\alpha_{1}=0.01 \in\left(0, \alpha_{1, \mathrm{cr}}\right), \quad \alpha_{2}=0.05 \in\left(\alpha_{1, \mathrm{cr}}, \alpha_{2, \mathrm{cr}}\right), \quad \alpha_{3}=0.1 \in\left(\alpha_{2, \mathrm{cr}}, 0.15\right) .
$$

The evolution of $s(t)$ in these three cases confirms the stability/instability character of the equilibria shown in the lower right panel of Fig. 4. 

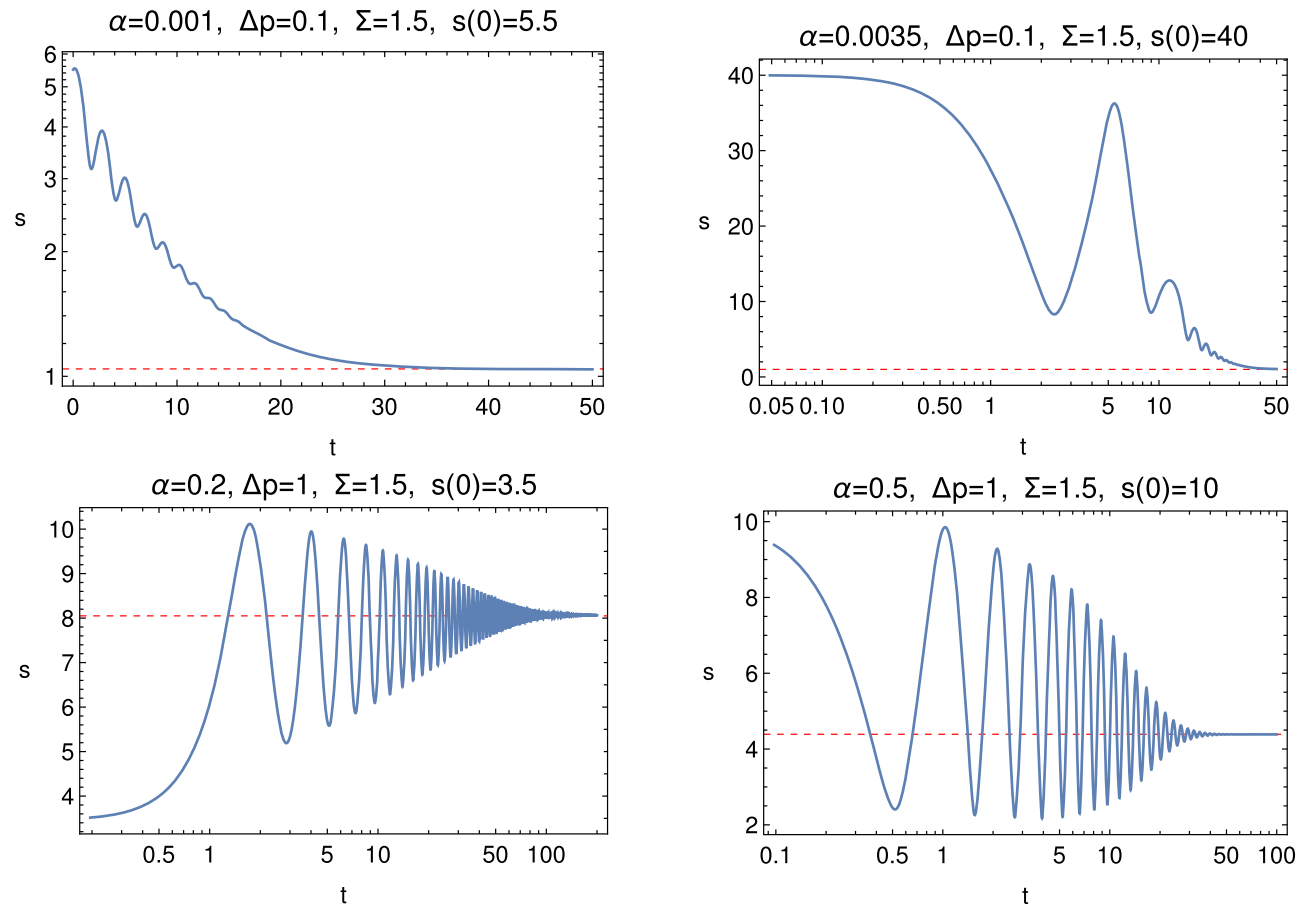

FIG. 3. Example of solutions which originate close to equilibria. In all cases, we fixed $a=3.5$ and $b=1$. Left upper panel, $s_{\text {eq }}=1.0473$. Right upper panel, $s_{\text {eq }}=1.147$. Lower left panel, $s_{\text {eq }}=8.01$. Lower right panel, $s_{\text {eq }}=4.39$

\section{Dynamics}

The general problem, that is $(31),(32)_{2}$, besides being highly nonlinear, is also of integral-differential type, namely

$$
\begin{gathered}
\ddot{s} s\left(1-\frac{s}{\sigma}\right)=-\dot{s}^{2}\left(\frac{3}{2}-2 \frac{s}{\sigma}+\frac{1}{2} \frac{s^{4}}{\sigma^{4}}\right)+ \\
+\Delta p+f+2 \frac{k_{0}}{G} e^{-b t} \ln \left(\frac{\sigma}{s}\right)-\frac{4 a\left(\Sigma^{3}-1\right)}{s^{3} \sigma^{3}} e^{-b t} \int_{0}^{t} \dot{s} s^{2} e^{b \tau} \mathrm{d} \tilde{\tau} .
\end{gathered}
$$

In particular, the initial data and $\Delta p$ are linked to $k_{0} / G$. Indeed, by setting $\ddot{s}_{o}=\ddot{s}(0), \dot{s}_{o}=\dot{s}(0)$, $s_{o}=s(0) \geq 1, f_{o}=f\left(s_{o}\right)$, and $\sigma_{o}=\sqrt[3]{\Sigma^{3}-1+s_{o}^{3}}$, we have

$$
2 \frac{k_{0}}{G} \ln \left(\frac{\sigma_{o}}{s_{o}}\right)=\ddot{s}_{o} s_{o}\left(1-\frac{s_{o}}{\sigma_{o}}\right)+\dot{s}_{o}^{2}\left(\frac{3}{2}-2 \frac{s_{o}}{\sigma_{o}}+\frac{1}{2} \frac{s_{o}^{4}}{\sigma_{o}^{4}}\right)-\Delta p-f_{o} .
$$

However, (38) can be rearranged as a third-order non-autonomous ODE. Indeed, multiplying by $s^{3} \sigma^{3} e^{b t}$, we obtain

$$
A_{1}(s, t) \ddot{s}=A_{2}(s, \dot{s}, t)-4 a\left(\Sigma^{3}-1\right) \int_{0}^{t} \dot{s} s^{2} e^{b \tau} \mathrm{d} \tau
$$

where

$$
A_{1}(s, t)=s^{4} \sigma^{3}\left(1-\frac{s}{\sigma}\right) e^{b t}
$$



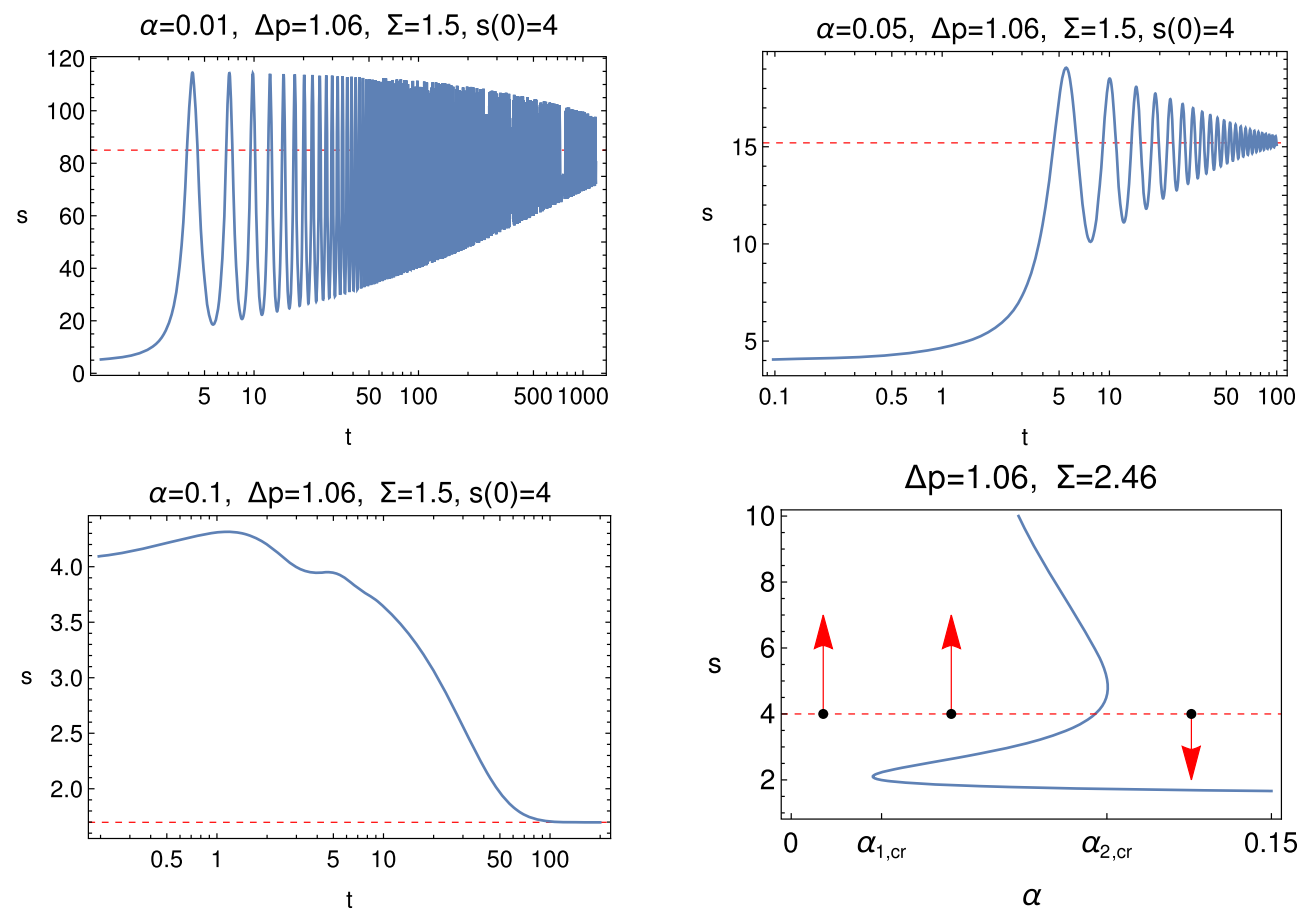

Fig. 4. The upper panels and the left lower panel show three solutions which originate in $s(0)=4$ for three different materials. The three values of $\alpha$ are chosen in such a way to be the first in $\left(0, \alpha_{1, \mathrm{cr}}\right)$, the second in $\left(\alpha_{1, \mathrm{cr}}, \alpha_{2}\right.$,cr $)$, and the third in $\left(\alpha_{2, \mathrm{cr}}, 0.15\right)$. Only the third solution is attracted toward an equilibrium close to $1\left(s_{\mathrm{eq}}=1.698\right)$, while the other two tend to stable but nonphysical equilibria $\left(s_{\text {eq }}=15.2\right.$ and $s_{\text {eq }}=85$, respectively). In all cases, $a=3.5$ and $b=1$

$$
A_{2}(s, \dot{s}, t)=s^{3} \sigma^{3}\left[\left(-\dot{s}^{2}\left(\frac{3}{2}-2 \frac{s}{\sigma}+\frac{1}{2} \frac{s^{4}}{\sigma^{4}}\right)+\Delta p+f\right) e^{b t}+2 \frac{k_{0}}{G} \ln \left(\frac{\sigma}{s}\right)\right],
$$

with $k_{0}$ given by (39). Now, it suffices to differentiate (40) once more with respect to time to get the following Cauchy problem

$$
\left\{\begin{array}{l}
A_{1} \dddot{s}=-\dot{A}_{1} \ddot{s}+\dot{A}_{2}-4 a\left(\Sigma^{3}-1\right) \dot{s} s^{2} e^{b t} \\
s(0)=s_{o} \geq 1, \dot{s}(0)=\dot{s}_{o}, \quad \ddot{s}(0)=\ddot{s}_{o}
\end{array}\right.
$$

where $A_{1}>0$.

Figure 5 shows some solutions to (41). In our simulations, we fixed $\Sigma=1.02, \alpha=0.5, a=b=1$, $s(0)=s_{o} \geq 1$, and $\dot{s}(0)=\ddot{s}(0)=0$. The pressure difference $\Delta p$ is allowed to vary from 0.01 to 1.5. It should be said, anyway, that numerical simulations show an extreme sensitivity to the remaining parameters, and physically significant bounded solutions can be obtained only for particular values of $\alpha$, $\Delta p$ and $\Sigma$. Any of these solutions can easily become singular for a small change of these values.

\section{Concluding remarks}

The problem of inflation of a nonlinear viscoelastic thick-walled spherical shell has numerous applications ranging from biology to space technology. However, the ability of a model to properly describe the peculiar dynamics of shells substantially resides in the reliability of the constitutive model. In this paper, we have tested the model illustrated in [13] and applied it to the problem of the oscillations of 

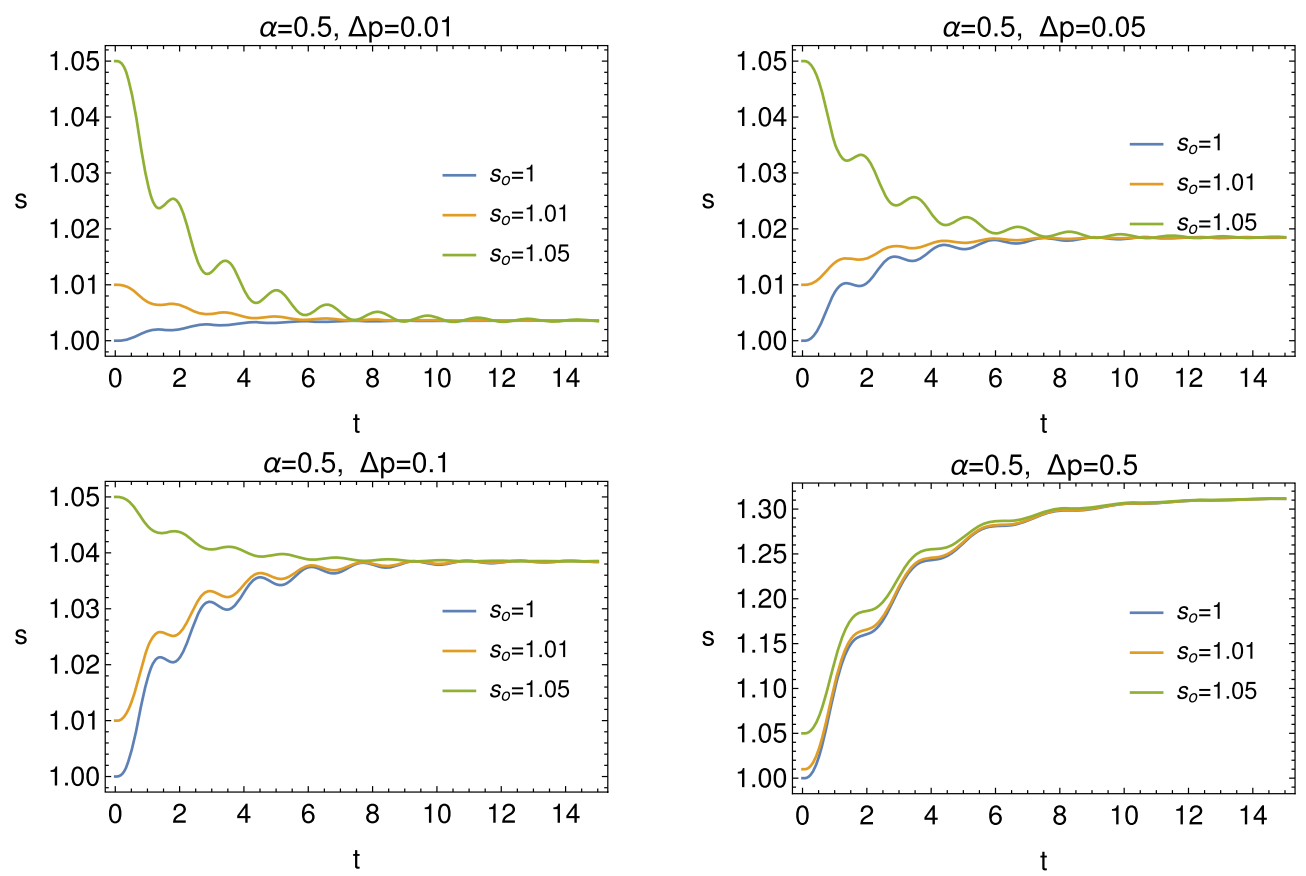

FIG. 5. For fixed $\alpha$, we simulate the dynamics governed by system (41). For identical choices of the initial value, the increase of $\Delta p$ leads, as expected, to the increase in asymptotic solution. Also, the oscillation frequency appears to decrease for increasing $\Delta p$

a spherical shell driven by an inner-outer pressure difference. The model predicts the existence of stationary configurations with spherical symmetry. The stability of these configurations has been analyzed considering only spherically symmetric disturbances, and therefore, the results obtained cannot be global. However, limiting ourselves only to spherically symmetric deformations, it turns out that the stationary configurations corresponding to isolated minima of the potential energy $V$ given by (35) are stable. The dynamics of the shell obeys to an integro-differential equation. Hence, the latter has been reduced to a non-autonomous third-order ordinary differential equation whose solution is decidedly easier. The numerical simulations have highlighted how the material parameters strongly influence the decay toward the stable configurations.

We believe that our model, besides being able to predict both stress relaxation and creep/recovery phenomena, has the advantage of a greater simplicity. Indeed, the comparison with other constitutive equations has to be read not just at a mathematical level, but rather considering the minimal number of parameters to be experimentally fitted.

\section{Acknowledgements}

This research was partially supported by G.N.F.M. of Italian I.N.D.A.M. and P.R.I.N. Project 2017KL4EF3_003, "Mathematics of active materials: from mechanobiology to smart devices". We express our sincere gratitude to the unknown referee for his/her useful suggestions and comments aimed at improving the quality of the paper.

Open Access. This article is licensed under a Creative Commons Attribution 4.0 International License, which permits use, sharing, adaptation, distribution and reproduction in any medium or format, as long as you give appropriate credit to the 
original author(s) and the source, provide a link to the Creative Commons licence, and indicate if changes were made. The images or other third party material in this article are included in the article's Creative Commons licence, unless indicated otherwise in a credit line to the material. If material is not included in the article's Creative Commons licence and your intended use is not permitted by statutory regulation or exceeds the permitted use, you will need to obtain permission directly from the copyright holder. To view a copy of this licence, visit http://creativecommons.org/licenses/by/4.0/.

Publisher's Note Springer Nature remains neutral with regard to jurisdictional claims in published maps and institutional affiliations.

\section{References}

[1] Luo, A.C.J.: Nonlinear Deformable-body Dynamics. Nonlinear Physical Science. Springer, Berlin (2010)

[2] Truesdell, C., Noll, W.: The non-linear field theories of mechanics, Vol. Handbuch der Physik Vol. III/3, Springer (1965)

[3] Calderer, M.C.: Radial motions of viscoelastic shells. J. Differ. Equ. 63, 289-305 (1983)

[4] Pucci, E., Saccomandi, G.: Some remarks about a simple history dependent nonlinear viscoelastic model. Mech. Res. Commun. 68, 70-76 (2015)

[5] Fosdick, R., Yu, J.H.: Thermodynamics, stability and non-linear oscillations of viscoelastic solids-ii. history type solids. Int. J. Non-Linear Mech. 33, 165-188 (1968)

[6] Gleysteen, J.J.: A history of intragastric balloons. Surg. Obes. Relat. Dis. 12(2), 435-450 (2016)

[7] De Pascalis, R., Parnell, W.J., Abrahams, I.D., Shearer, T., Daly, D.M., Grundy, D.: The inflation of viscoelastic balloons and hollow viscera. Proc. R. Soc. A 474(20180102), 1-22 (2018)

[8] Pipkin, A.C., Rogers, T.G.: A non-linear integral representation for viscoelastic behaviour. J. Mech. Phys. Solids 16, 59-72 (1968)

[9] De Pascalis, R., Napoli, G., Saccomandi, G.: Kink-type solitary waves within the quasi-linear viscoelastic model. Wave Motion 86, 195-202 (2019)

[10] Zhou, Z.: Creep and stress relaxation of an incompressible viscoelastic material of the rate type. Int. J. Solid Struct. 28(5), 617-630 (1991)

[11] Filograna, L., Racioppi, M., Saccomandi, G., Sgura, I.: A simple model of nonlinear viscoelasticity taking into account stress relaxation. Acta Mech. 204(1-2), 21-36 (2009)

[12] Saccomandi, G., Vianello, M.: Shear waves in a nonlinear relaxing media: a three-dimensional perspective. J. Acoust. Soc. Am. 149, 1589-1595 (2021)

[13] Farina, A., Fusi, L., Rosso, F., Saccomandi, G.: Creep, recovery and vibration of an incompressible viscoelastic material of the rate type: simple tension, Int. J. Nonlinear Mech. 138: 103851. https://doi.org/10.1016/j.ijnonlinmec.2021.103851

[14] Maccamy, R.C.: Existence, uniqueness and stability of solutions of the equation $u_{t t}=\frac{\partial}{\partial x}\left(\sigma\left(u_{x}\right)+\lambda\left(u_{x}\right) u_{t t}\right)$. Indiana Univ. Math. J. 20, 231-238 (1970)

[15] Jaunzemis, W.: Continuum Mechanics. Macmillan, New York (1967)

[16] Truesdell, C., Rajagopal, K.R.: An Introduction to the Mechanics of Fluids. Birkhäuser, Basel (2000)

[17] Rajagopal, K.R.: Remark on the notion of "pressure". Int. J. Non-Linear Mech. 71, 165-172 (2015)

[18] Phan-Thien, N., Safari-Ardi, M., Morales-Patino, A.: Oscillatory and simple shear flows of a flour-water dough: a constitutive model. Rheol. Acta 36, 38-48 (1997)

[19] Nasseri, S., Bilston, L.E., Phan-Thien, N.: Viscoelastic property of pig kidney in shear, experimental results and modelling. Rheol. Acta 41, 180-192 (2002)

[20] Calderer, C.: The dynamical behaviour of nonlinear elastic spherical shells. J. Elast. 13, 17-47 (1983)

[21] Parter, S.V., Rajagopal, K.R.: Swirling flow between rotating plates. Arch. Ration. Mech. Anal. 86, 305-315 (1984)

[22] Rajagopal, K.R.: Flow of viscoelastic fluids between rotating disks. Theoret. Comput. Fluid Dyn. 3, 185-206 (1992)

[23] Hurwitz, A.: Über die Bedingungen, unter welchen eine Gleichung nur Wurzeln mit negativen reellen Teilen besitzt. Math. Ann. 46, 273-284 (1895)

[24] Fuller, A.T.: Conditions for a matrix to have only characteristic with negative real parts. J. Math. Anal. Appl. 23, 71-98 (1968) 
Angiolo Farina, Lorenzo Fusi and Fabio Rosso

Dipartimento di Matematica e Informatica "Ulisse Dini"

Università degli Studi di Firenze

Firenze

Italy

e-mail: angiolo.farina@unifi.it

Lorenzo Fusi

e-mail: lorenzo.fusi@unifi.it

Fabio Rosso

e-mail: fabio.rosso@unifi.it

Giuseppe Saccomandi

Dipartimento di Ingegneria

Università di Perugia

Perugia

Italy

e-mail: giuseppe.saccomandi@unipg.it

(Received: November 8, 2021; revised: January 25, 2022; accepted: January 29, 2022) 